\title{
Assessment of the psychological status of children with growth hormone deficiency and their parents
}

\author{
K S H de Silva ${ }^{1}$, P de Zoysa ${ }^{2}$ \\ (Index words: growth hormone deficiency, psychological status, Sri Lankan children)
}

\begin{abstract}
Introduction Children with growth hormone deficiency may have psychological ramifications due to their disease condition. Having a child with growth hormone deficiency may affect the parents' psychological health.

Objectives To assess the psychological health of children with GHD and their parents.

Methods A descriptive cross sectional study was done over four years from July 2006 on children with growth hormone deficiency. The self-administered, culturally validated Personality Assessment Questionnaire (PAQ) was given to children above 8 years of age. By considering seven personality dimensions, the $P A Q$ assesses whether a child is psychologically maladjusted. A score $\geq 89$ indicates such maladjustment. The parents were given the self-administered General Health Questionnaire-30 (GHQ-30). A score $\geq 4$ is indicative of mental health difficulties in the respondent.

Results Fourty of 56 children with GHD were more than 8 years of age and there were 33 boys. According to the PAQ, 24 children (60\%: 19 boys) appeared to be psychologically maladjusted. Of the 74 parents (47 mothers) who answered the GHQ-30, forty (54\%) were deemed to have mental health difficulties, a majority of them were mothers.
\end{abstract}

Conclusions Psychological maladjustment was observed in a majority of children in the study and most of their mothers had mental health difficulties.

Ceylon Medical Journal 2011; 56: 59-61

\section{Introduction}

Growth hormone $(\mathrm{GH})$ is not essential for life but is essential for growth in height. Having an extremely short child due to GHD has a great impact on the whole family and, needless to say, the burden is far greater on the child.

Though a certain amount of research has been done in other countries on the effects of GHD, such research is scarce in Sri Lanka. This study is a preliminary investigation on the psychological effects of living with GHD. Specifically it was hypothesised that a child with GHD may have psychological maladjustment, and that having such a child would have an impact on the parents' psychological health.

\section{Methods}

A descriptive, cross sectional study was carried out on children with GHD followed up at the University Paediatric Unit of the Lady Ridgeway Hospital, Colombo (LRH) for 4 years from July 2006 to July 2010. The sample included all willing children over 8 years of age and their parents who attended the clinic during the study period. The questionnaires were administered once the diagnosis of GHD was confirmed and before commencement of therapy. Ethics approval for the study was obtained from the Ethics Review Committee of the Faculty of Medicine, Colombo.

Subsequent to obtaining verbal consent, the PAQ and GHQ-30 (30 items) were self-administered on the children and parents respectively. The test administrator was present throughout to clarify any doubts during the testing procedure. Psychological maladjustment of the children was assessed by the culturally validated Sinhala version of the PAQ which has shown good psychometric properties and has been used in previous research [1]. The PAQ was also translated to Tamil, specifically for this study. The questionnaire assesses whether a child is psychologically maladjusted. This is measured in 7 dimensions: hostility and aggression, dependency, negative self-esteem, negative self-adequacy, emotional unresponsiveness, emotional instability and negative world view. It has 42 items with 4 responses each. The response options are: 'almost always true of me', 'sometimes true of me', 'rarely true of me' and 'almost never true of me' where the respondent selects the most appropriate answer describing himself/herself with regard to each item. Fourteen items are reverse scored to prevent 'blind' answering. A score at or above 89 is indicative of psychological maladjustment in the child [1].

The mental health of the parents was assessed by the self-administered GHQ-30 which has been widely used both internationally and in Sri Lanka. This 30 item questionnaire has four response options. These are: 'better than usual', 'same as usual', 'less than usual' and 'much less than usual'. A score at or above 4 identifies the respondent as having mental health difficulties [2]. 


\section{Results}

There were 56 children with GHD, of whom 40 were above 8 years of age and eligible for the study. Thirty three were boys. A significant score at or above 89 on the PAQ was obtained from 24(60\%) children (Table1). Seventy four parents (63.5\% mothers) answered the GHQ30 . A significant score at or above 4 indicating the presence of mental health difficulties was found in 40 (54\%) of them, of which 28 (70\%) were mothers (Table 2).

Table 1. Assessment of children by the PAQ $(n=40)$

\begin{tabular}{lllc}
\hline Score & Boys & Girls & $\begin{array}{c}\text { Total number } \\
(\%)\end{array}$ \\
\hline$\geq 89$ & 19 & 5 & $24(60)$ \\
$<89$ & 14 & 2 & $16(40)$ \\
Total (\%) & $33(82.5)$ & $7(17.5)$ & $40(100)$ \\
\hline
\end{tabular}

PAQ - Personality Assessment Questionnaire

Table 2. Assessment of parents by the GHQ-30 $(n=74)$

\begin{tabular}{lllc}
\hline Score & Mothers & Fathers & $\begin{array}{c}\text { Total number } \\
(\%)\end{array}$ \\
\hline$\geq 4$ & 28 & 12 & $40(54)$ \\
$<4$ & 19 & 15 & $34(46)$ \\
Total (\%) & $47(63.5)$ & $27(36.5)$ & $74(100)$ \\
\hline
\end{tabular}

GHQ-30 - General Health Questionnaire-30

\section{Discussion}

GHD is characterised by a short child, who if untreated, will grow up to be an extremely short adult. The incidence of GHD in Sri Lanka is not known. Worldwide incidence is documented as 1 in 4000 and hence is not a common condition. Of the 33 boys answering the PAQ, 19 $(57.6 \%)$ were deemed to be psychologically maladjusted. There were only 7 girls who fulfilled the inclusion criteria and almost all of them had a significant score on the PAQ, indicating psychological maladjustment.

This study highlights that GHD is associated with psychological maladjustment in children. Though a causal pathway is not indicated by these results, it may indicate that timely and appropriate treatment for GHD may help overcome the psychological ramifications associated with this condition. A study from the United Kingdom assessing quality of life (QOL) in children with idiopathic GHD and acquired GHD at initiation of GH treatment and 6 months later demonstrated some QOL benefits of treatment and recommended comprehensive assessment of GH treatment to include QOL aspects [3]. An Italian study on adults with untreated childhood onset GHD demonstrated a definite improvement in several aspects of psychological and psycho-neuro-physiological functioning after 6 months of treatment with recombinant $\mathrm{GH}$ [4].

Our patients were assessed only prior to therapy as they have had treatment for variable periods of time, as an uninterrupted free supply of GH is yet to be established at the LRH. Such a supply would be important not only to address the primary concern of GHD but also to combat its potential psychological ramifications. However, certain studies have shown that patients successfully treated with GH in childhood, have reported poor QOL and symptoms of certain psychiatric disorders during young adulthood 5 . Hence it appears that irrespective of treatment, GHD is associated with a patient's psychological functioning in a complex, non-linear manner.

A further finding from this study was that a majority of parents of children with GHD showed evidence of mental health difficulties. Though these results are preliminary and conclusions on causality cannot be made, this finding is not surprising as having a child with a disease condition that has an impact on his/her quality of life would indeed be a psychological burden on the parents. This may be particularly so in traditional and folk-lore based cultures such as Sri Lanka where the populace, mostly those in rural areas, may consider extreme shortness as a symbol of inauspiciousness, thus affecting the child and family. The results also showed that a greater number of mothers had mental health difficulties than fathers. This result too is not surprising as mothers, particularly in traditional societies such as Sri Lanka, take on more family care giving roles than fathers.

Though this study is the first on Sri Lankan children with GHD and their psychological ramifications, it is not without limitations. The small sample size is one such limitation. Hence the generalisability and the statistical power of the research are limited. In respect of the measures investigated in this study, only global measures of psychological dysfunction were explored. Future research may need to focus on particular types of psychological difficulties such as the impact of GHD on self esteem and self confidence. The questionnaires used were translated to Sinhala and Tamil and have been used in other crosscultural research, but the GHQ-30 and the Tamil PAQ have not been validated in Sri Lanka. Despite the above limitations, this preliminary study offers an important insight into the psychological functioning of children with GHD and their parents.

\section{Acknowledgements}

We wish to thank Drs N. Joseph and G. R. Suranga for their assistance with the preliminary work leading up to the study. 


\section{References}

1. De Zoysa P, Rajapakse LS, Newcomb PA. Adaptation and validation of the Personality Assessment Questionnaire on children in Sri Lanka. LS Boyar ed, 2007; New psychological tests and testing research. New York: Nova Science Publishers, Inc.

2. Goldberg DP. The detection of psychiatric illness by questionnaire. Mudsley Monograph No. 21; 1972 Oxford University Press. London, UK.

3. Sheppard L, Eiser C, Davies HA, et al. The effects of growth hormone treatment on health-related quality of life in children. Hormone Research 2006; 65: 243-9.

4. Sartorio A, Molinari E, Riva G, Conti A, et al. Growth hormone treatment in adults with childhood onset growth hormone deficiency: effects on psychological capabilities. Hormone Research 1995; 44: 6-11.

5. Stabler B. Impact of growth hormone $(\mathrm{GH})$ therapy on quality of life along the lifespan of GH-treated patients. Hormone Research in Paediatrics 2001; 56: 55-8. 